\title{
Desarrollo de recursos didácticos con el uso de las Tecnologías de la información y comunicación disponibles en una plataforma virtual
}

\author{
Development of didactic resources with the use of information and communication \\ technologies available on a virtual platform
}

DEL CARMEN-MORALES, Yucels Anaíi*, DEL CARMEN-MORALES, Heidi, FELIPEREDONDO, Ana María y HERNÁNDEZ-BAUTISTA, Anayeli

Universidad Tecnológica de la Huasteca Hidalguense

ID $1^{\text {er }}$ Autor: Yucels Anaí, Del Carmen-Morales / ORC ID: 0000-0003-2738-4780, Researcher ID Thomson: I-6613-2018, CVU CONACYT ID: 905179

ID $1^{\text {er }}$ Coautor: Heidi, Del Carmen-Morales / ORC ID: 0000-0002-9686-1838, Researcher ID Thomson: O6682-2018, CVU CONACYT ID: 926525

ID $2^{\text {do }}$ Coautor: Ana María, Felipe-Redondo / ORC ID: 0000-0002-8579-6532, CVU CONACYT ID: 835952

ID $3^{\text {er }}$ Coautor: Anayeli, Hernández-Bautista / ORC ID: 0000-0002-5621-2585

DOI: $10.35429 / J I T .2019 .19 .6 .28 .36$

Recibido: 12 de Marzo, 2019; Aceptado 30 de Junio, 2019

Resumen

Desarrollar recursos didácticos con el uso de las Tics para fortalecer la enseñanza de las matemáticas en los estudiantes de la Universidad Tecnológica de la Huasteca Hidalguense fue la finalidad de este estudio. Los resultados de la evaluación diagnostica aplicada a una muestra de 856 alumnos de nuevo ingreso indican que el desempeño es regular (6.1 puntos) en Aritmética e insuficiente (4.4) en Algebra (Academia de matemáticas UTHH,2018); proyecta las siguientes problemáticas: índices de reprobación, deserción, el temor a los cálculos matemáticos y la solución de problemas relacionados a su campo de aplicación, por lo que se propone el desarrollo de recursos didácticos disponibles en una plataforma accesible. Según (CONOCER, 2016) los recursos didácticos son instrumentos utilizados en el contexto educativo mediante su manipulación, observación, o lectura, se ofrezcan oportunidades de aprender o con su uso se intervenga en el desarrollo de alguna función de la enseñanza; (Spiegel, 2006) indica son necesarios para promover la autonomía de los alumnos, futuros trabajadores en el perfil que enseñamos. Este trabajo está basado en la Metodología para el desarrollo de aplicaciones educativas en ambientes multimedios (Bianchini, 1992) con las fases: a) Investigación y Análisis; b) Diseño lógico, funcional y físico; c) desarrollo; d) Implantación y producción. Como resultado se obtuvieron recursos didácticos (videos, infografías y evaluaciones digitales) que apoyen el proceso de enseñanza aprendizaje mediante información organizada que complemente, amplié y detalle procesos de solución para docentes y alumnos disponible en una plataforma virtual institucional.

Matemáticas, Recursos, Ambientes virtuales, Multimedia

\begin{abstract}
Develop didactic resources with the use of ICT'S (Information Communication Tecnologies) to strengthen mathematics teaching with students of the Technological University of Huasteca Hidalguense it was the purpose of this study. The results of the diagnostic evaluation applied to a sample of 856 new students indicate that the performance is regular (6.1 points) in Arithmetic and insufficient (4.4) in Algebra (UTHH Academy of Mathematics, 2018); shows the following problems: failing rates, dropping out, mathematical fear calculations and problems solving related to their field of application, so that proposes the development of teaching resources available on an accessible platform. According to (CONOCER, 2016) the learning resources are instruments used in the educational context through their manipulation, observation, or reading, opportunities to learn are offered or with their use they intervene in the development of some teaching function; (Spiegel, 2006), It indicates they are necessary to promote the autonomy of students, future workers in the profile we teach. This work is based on the Methodology for the development of educational applications in multimedia environments (Bianchini, 1992) with the phases: a) Research and Analysis; b) Logical, functional and physical design; c) development; d) Implementation and production. As a result, educational resources were obtained (videos, infographics and digital evaluations) these support the teaching-learning process through organized information that complements, expands and details solution processes for teachers and students available on an institutional virtual platform.
\end{abstract}

Mathematics, Resources, Virtual environments, Multimedia

Citación: DEL CARMEN-MORALES, Yucels Anaí, DEL CARMEN-MORALES, Heidi, FELIPE-REDONDO, Ana María y HERNÁNDEZ-BAUTISTA, Anayeli. Desarrollo de recursos didácticos con el uso de las Tecnologías de la información y comunicación disponibles en una plataforma virtual. Revista de Tecnologías de la Información. 2019. 6-19: 28-36

$\dagger$ Investigador contribuyendo como primer autor. 


\section{Introducción}

La incorporación de las Tecnologías de la Información y la Comunicación (TIC) en el ámbito de la educación, aportan recursos y herramientas didácticas que impactan en la enseñanza y el aprendizaje de los estudiantes, proporcionado un espacio de comunicación, asesorías y formación. Tomando como referencia los resultados de la evaluación diagnostica aplicada a una muestra aplicada a 856 alumnos de nuevo ingreso, la academia de matemáticas de la UTHH, analiza que el desempeño es regular (6.1 puntos) en aritmética e insuficiente (4.4 puntos) en algebra (Academia de Matemáticas UTHH, 2018); de acuerdo al reporte de índices de deserción, reprobación ocupa el 51.9\%, problemas económicos el 7.4\%, deserción si causa conocida el $8.1 \%$, incumplimiento de expectativas el $3.0 \%$, cambio de carrera $3.7 \%$, problemas personales $5.9 \%$, cambio de UT $5.2 \%$ otras causas $14.8 \%$, faltas al reglamento escolar 4.3\%. (UTHH, Estadistica basica institucional , 2019).

Sumado a esta problemática el temor de los estudiantes a los cálculos matemáticos, la solución de problemas y trabajo colaborativo, son aspectos que los docentes enfrentan en las aulas en el quehacer educativo e impactan de manera significativa son los índices de reprobación por lo que se propone el desarrollo de recursos didácticos con la finalidad de fortalecer el proceso de enseñanza aprendizaje de las ciencias básicas. En base a lo antes mencionado este trabajo de investigación tiene como finalidad "desarrollar recursos didácticos mediante el uso de las Tecnologías de la Información y la Comunicación (TIC) para fortalecer la enseñanza de las matemáticas en los estudiantes de la Universidad Tecnológica de la Huasteca Hidalguense.

\section{Fundamentos Teóricos}

Importancia de las TICS en la enseñanza a nivel superior:

Según (Ponce, 2006)Las TIC son todas las herramientas utilizadas tanto en colegios, como en empresas, que ayudan a transmitir información, además de ser utilizadas como herramientas de trabajo para la construcción de conocimiento, sobre todo en el transcurso de las etapas educativas.
Las Tecnologías de la Información y la Comunicación tienen un potencial reconocido para apoyar el aprendizaje, el conocimiento y el desarrollo de habilidades y competencias para aprender autónomamente. Esto sucede porque las TIC ayudan a la motivación del estudiante, la capacidad de resolver problemas, mejora el trabajo en grupo, refuerza la autoestima del alumno al desarrollar la autonomía de aprendizaje, además de tener la ventaja de poder acceder a ellas desde cualquier parte y a cualquier hora.

\section{Recursos didácticos}

Según (CONOCER, 2016) los recursos didácticos son instrumentos utilizados en el contexto educativo mediante su manipulación, observación, o lectura, se ofrezcan oportunidades de aprender o con su uso se intervenga en el desarrollo de alguna función de la enseñanza; (Spiegel, 2006), indica son necesarios para promover la autonomía de los alumnos, futuros trabajadores en el perfil que enseñamos.

\section{Aplicaciones de los Recursos Didácticos:}

De acuerdo a (Spiegel, 2006) La selección y la elaboración de los recursos didácticos están determinadas por la secuencia de didáctica del módulo. Por otra parte, no existe un recurso que solucione "todo" y que sea útil para "todas" las circunstancias de la clase ni para todas las clases. Así, los recursos son valiosos a partir de que se les reconoce una ventaja o un aporte específico para:

\footnotetext{
- El desarrollo de las capacidades del módulo.

- Cumplir la función asignada por la secuencia didáctica;

El contexto de la clase (sus alumnos, el ámbito en el que se desarrollara, etc.).
}

Una vez seleccionados o elaborados por el docente, constituirán sus herramientas de trabajo; con ella llevara a cabo las actividades tendientes a facilitar la construcción de las capacidades relacionadas con el problema de la práctica profesional que el participante debiera ser capaz de resolver al finalizar el modulo.

El uso de los recursos didácticos en el aula son importantes porque: 


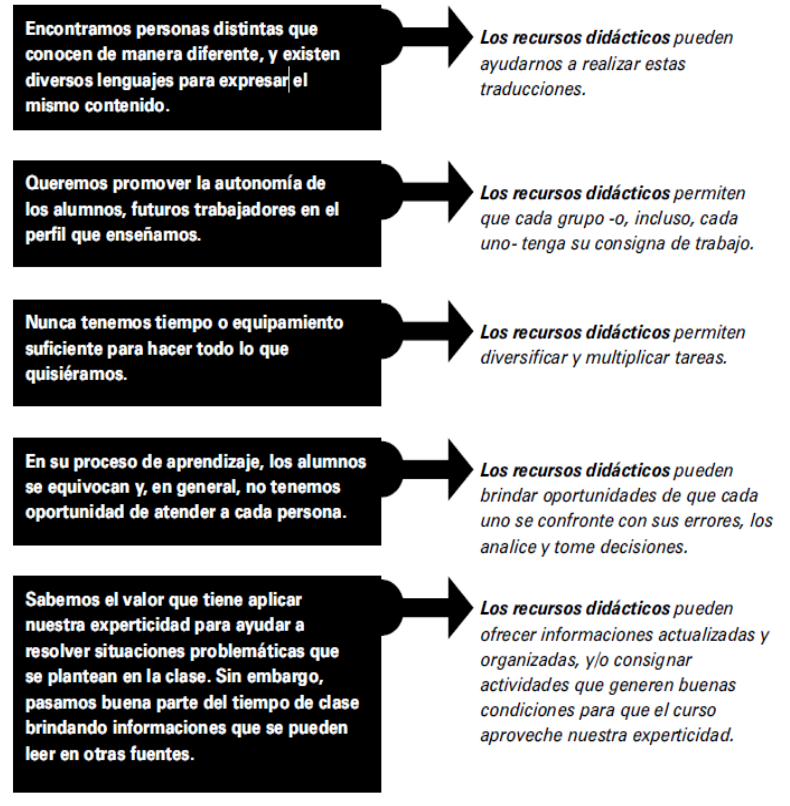

Figura 1 En la siguiente imagen se muestra el uso de los recursos didácticos

Fuente: (Spiegel, 2006)

Los recursos didácticos son usados tomando en cuenta la secuencia didáctica del módulo y las características concretas de la clase que se está preparando

\section{Clasificación de los Recursos Didácticos:}

Existen diferentes clasificaciones de los recursos didácticos o medios de enseñanza, sin embargo, convencionalmente pueden dividirse en cuatro subgrupos, según (Cañedo, 2000):

- $\quad$ Objetos naturales e industriales, pueden tener su forma normal (animales vivos y disecados, herbarios, colecciones entomológicas y de minerales, máquinas industriales, agropecuarias, etc.), o presentarse cortadas en sección, a fin de mostrar su estructura interna.

Objetos impresos y estampados, Se confeccionan de forma plana, laminas, tablas, gráficos, guías metodológicas, libros y cuadernos, etc., así como también medios tridimensionales representativos, como modelos, maquetas, etc.,

Medios sonoros y de proyección.

Se subdividen en audiovisuales: películas y documentales didácticos, sonoros y videocintas; visuales: fílmicas y diapositivas; y auditivos: grabaciones magnetofónicas en placas o discos.

Materiales para la enseñanza programada y de control,
Pueden ser, atendiendo a su estructura, lineales, ramificados, y mixtos. En este último subgrupo se incluyen los llamados medios de programación y de control, materializados a través de diferentes softwares educativos y las destinadas a controlar la adquisición de conocimientos - Transmisión de la información, como la película didáctica, el libro de texto y materiales de Internet.

La experimentación escolar, como los equipos, utensilios e instrumentos de laboratorio $\cdot$ Los de entrenamiento, que agrupa a los simuladores y a otros equipos que se emplean para reproducir situaciones que requieren habilidades manipulativas El uso adecuado de los medios de enseñanza eleva las posibilidades y la calidad del trabajo de los profesores, o sea, su eficacia metodológica-pedagógica, y perfecciona las actividades cognoscitivas y de asimilación de los estudiantes, en las diferentes etapas del proceso de enseñanza. aprendizaje. (Cañedo \& Cáceres, 2014)

\section{Tipos de Recursos Didácticos:}

Infografías digitales

Es un diseño gráfico en el que se combinan textos y elementos visuales con el fin de comunicar información precisa sobre variadas temáticas (científicas, deportivas, culturales, literarias, etc.). Este recurso aproxima al lector a los elementos, ideas o acontecimientos más importantes de un determinado tema, como: dónde ocurrió, cómo se llevó a cabo, cuáles son sus características, en qué consiste el proceso, cuáles son las cifras, etc. Las infografías son tremendamente útiles y esenciales para representar la información que es complicada de entender a través del puro texto. Con un simple golpe de vista se puede entender hasta las cosas más complicadas, y además son más fáciles de asimilar y recordar. (Castro \& Colmenar)

\section{- Películas:}

Complementa y profundiza temas a estudiar en las asignaturas, anima a la discusión y ayuda a la comprensión de los contenidos, aquí el profesor juega el papel de orientador, moderador y mediador. 
Permite comprender aspectos relacionados y que forman parte de un todo que ayuda a entender la sociedad, las relaciones de poder, las mentalidades y cuestiones de la vida cotidiana. (Castro \& Colmenar)

- Juegos de simulación:

Los juegos de simulación son una herramienta que facilita el aprendizaje, ya que representa un modelo simplificado de la realidad. Se suelen crear a medida; para ello se estudian las principales características que se desean simular, se fija el objetivo que se pretende enseñar y luego se busca un mecanismo entretenido que se adapte a dichas condiciones. Puede ser un tablero, un programa de ordenador, etc. (Castro \& Colmenar)

Videos digitales:

Al hablar de vídeo digital nos estamos refiriendo en primera instancia a un proceso técnico que se aplica a cuatro momentos importantes de la manipulación de la información: Al capturar, al transmitir la información, al conservar y al reproducir. (Castro \& Colmenar)

Ambientes virtuales de aprendizaje:

(Stiles, 2000) indica que un " 'Ambiente Virtual de Aprendizaje' o 'Sistema Administrador del Aprendizaje' está diseñado para actuar como centro de las actividades de los estudiantes, para su administración y facilitación, junto con la disposición de los recursos requeridos para ellas", opcionalmente puede incluir un sistema que soporte la "la inscripción, las opciones administración de los cursos, carpeta y perfil del estudiante, un sistema de mensajería y publicación del contenido". un ambiente virtual de aprendizaje es equivalente a aún sistema de administración del aprendizaje (LMS por sus siglas en inglés).

Existe una amplia oferta de LMS, los hay de pago como WebCT, Blackboard o Lotus Learning Management System y de código abierto y libre distribución como Moodle, Interact, Claroline, Fle3, Manhattan entre muchos otros. Sin embargo y a pesar de la gran oferta todos ellos son una colección de herramientas para la comunicación y gestión de contenidos para la WWW.
Por ejemplo, la mayoría de los LMS integran herramientas para comunicación síncrona (chat y mensajería instantánea) y asíncrona (foros en web y correo electrónico), posibilidad de crear páginas con la información general de los usuarios, capetas de trabajo, calendario de actividades, sistemas de encuestas, sistema de bitácoras de actividad y un sistema de administración escolar. Bajo esta idea un LMS es una colección de herramientas que nos permiten administrar las actividades propuestas para los estudiantes, poner a disposición un sistema de comunicación efectivo y tener un mejor control escolar, por todo esto es mejor referirse a ellos como a Sistemas para la Administración del Conocimiento (SAC) (Miranda, 2004)

\section{Estadísticas de deserción UTHH:}

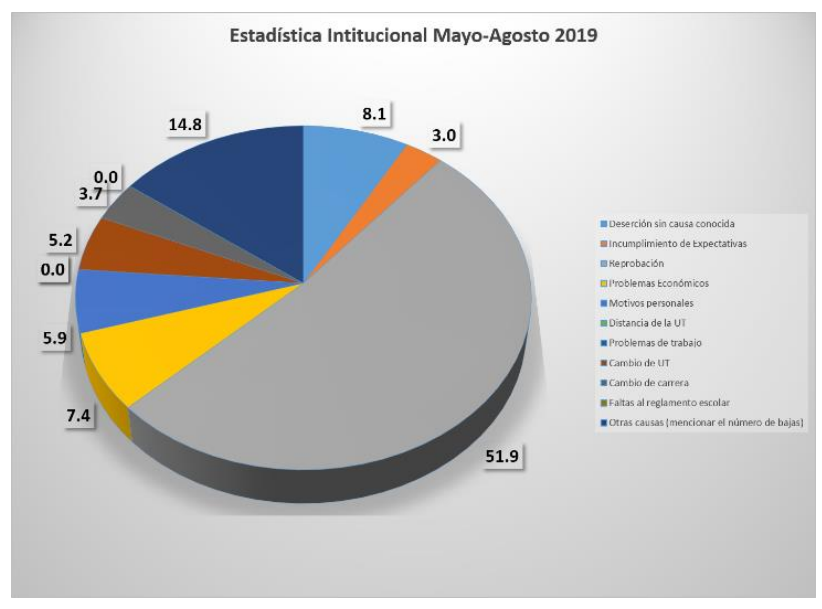

Figura 2 En la siguiente grafica se muestran los porcentajes de cada causa de deserción institucional en el alumnado de la UTHH

Fuente: (UTHH, Estadistica basica institucional , 2019)

Reporte de índices de deserción: reprobación ocupa el 51.9\%, problemas económicos el $7.4 \%$, deserción si causa conocida el $8.1 \%$, incumplimiento de expectativas el $3.0 \%$, cambio de carrera $3.7 \%$, problemas personales $5.9 \%$, cambio de UT $5.2 \%$ otras causas $14.8 \%$, faltas al reglamento escolar $4.3 \%$. (UTHH, Estadistica basica institucional, 2019).

\section{Diagnostico Aplicado por la Academia de matemáticas UTHH}

Se evaluó a 856 alumnos de las carreras de:

Evaluación de Proyectos

Desarrollo de negocios

Ingeniería en Agrobiotecnología.

Ingeniería en Financiera Fiscal y Contador público. 
Ingeniería en Tecnologías de la información Licenciatura en gastronomía

Ingeniería Civil

Ingeniería en procesos alimentarios Ingeniería en Metal Mecánica

Ingeniería en Mecatrónica.

Se aplicó un instrumento definido por la academia de matemáticas UTHH, realizando una evaluación inicial a los alumnos de nuevo ingreso periodo 2018-3 Mes de Agosto.

Se impartió un curso propedéutico de nivelación y se aplicó una evaluación Final.

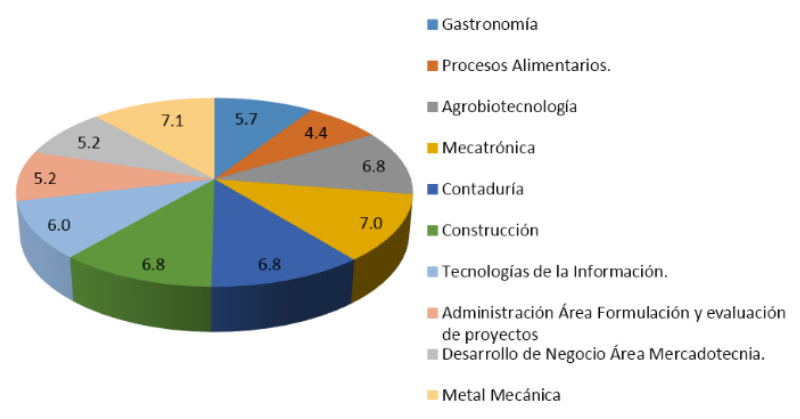

Figura 3 Resultados Evaluación inicial (Aritmética por programa educativo. Escala: 1-2 Deficiente 3-4 Insuficiente 5-6 Regular 7-8 Bueno 9-10 Excelente Fuente: (Academia de matemáticas, UTHH)

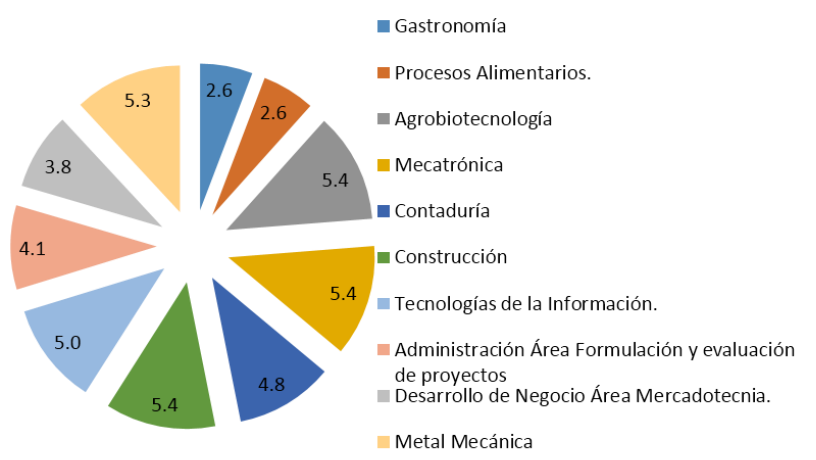

Figura 4 Resultados Evaluación Inicial (Algebra) Aritmética por programa educativo. Escala: 1-2 Deficiente 3-4 Insuficiente 5-6 Regular 7-8 Bueno 9-10 Excelente Fuente: (Academia de matemáticas, UTHH)

\section{Metodología}

Para la realización de los recursos didácticos matemáticos se utilizará la "Metodología para el desarrollo de aplicaciones educativas en ambientes multimedios" (Bianchini, 1992) la cual consta de cuatro fases:

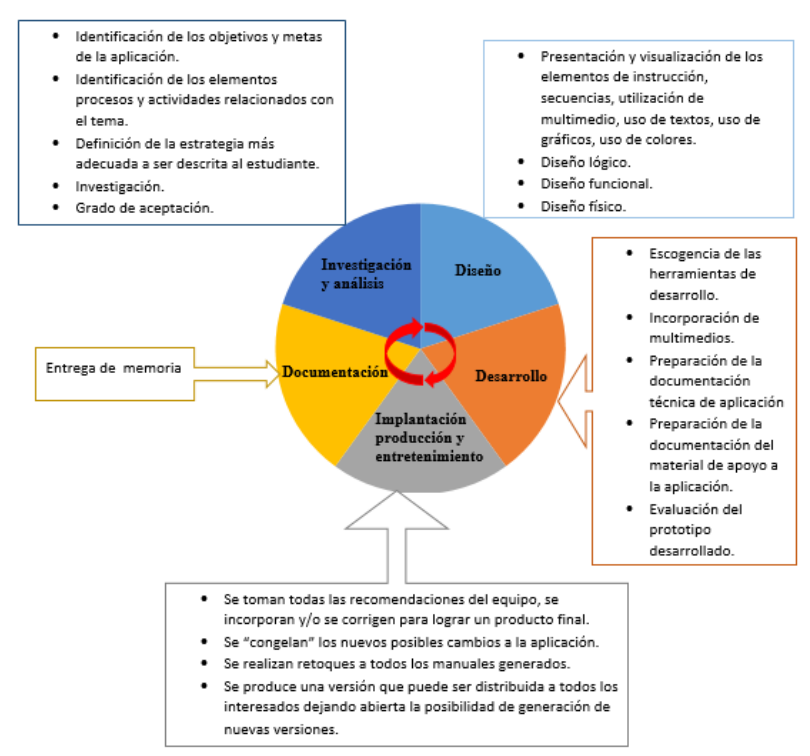

Figura 5 Metodología para el desarrollo de aplicaciones educativas

Fuente: (Bianchini, 1992)

\section{Fase I: Investigación y análisis}

En esta fase se definieron los propósitos y metas, elementos, procesos y actividades relacionados con el tema, las condiciones de uso, usuarios, la recopilación del conocimiento previo, definición de estrategias y material didáctico para el desarrollo del proyecto.

En reuniones de academia se determinó el desarrollo de infografías, guiones y videos para la materia de algebra lineal considerando las hojas de asignaturas; se definió que las secciones de cada tema serian: definición, área de aplicación, ejemplos, practicas, evaluación y bibliografía.

Se consideró la importancia de reunir estos recursos en un espacio accesible para el alumnado, ya que es fundamental para propiciar nuevos espacios de aprendizaje y colaboración por lo que se determinó que un medio eficiente seria la Plataforma Virtual UTHH donde se establecerá una interacción entre: Administradores, docentes y alumnos.

\section{Fase II: Diseño}

\section{a) Diseño lógico}

En esta imagen se muestra el mapa de navegación de la Plataforma virtual para los recursos didácticos de la materia de algebra. 
- $\quad$ En el apartado de definición permitirá agregar infografías de acuerdo al tema. Área de aplicación: en este apartado se describirán como es que los temas se utilizan en la vida cotidiana y la importancia en ella.

- $\quad$ Ejemplos: en este apartado se encuentran los videos realizados para que el alumno pueda retroalimentar el tema describiendo ejemplos relacionados con el entorno.

- Prácticas: aquí se encontrarán ejercicios para que el alumno retroalimente su aprendizaje.

- El sitio cuenta con un apartado de evaluación, esto permitirá que el alumno conteste un examen digital para medir su aprendizaje y de esta manera el docente se podrá dar cuenta en que temas debe reforzar al alumno.

- Mis cursos: Los docentes podrán subir los recursos didácticos tales como, videos, infografías y evaluaciones didácticas.

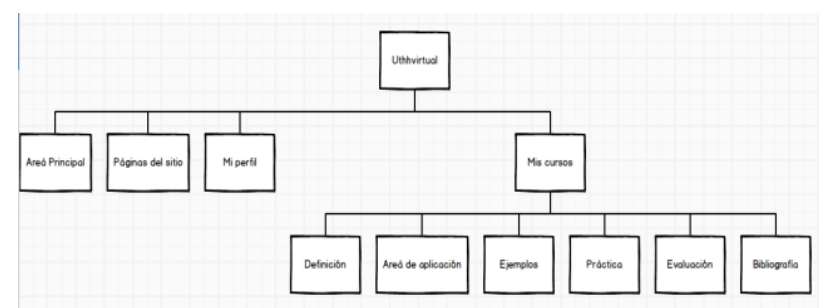

Figura 6 Mapa de navegación de la Plataforma virtual UTHH

Fuente: Elaboración Propia

b) Diseño functional

Se determinó la estandarización de imágenes, tipografía, videos e icono.

\begin{tabular}{|c|c|c|c|}
\hline Nombre & Icono & Tamaño & Utilidad \\
\hline Objetivos & (C) & $\begin{array}{l}\text { Height: } 16 \mathrm{px} . \\
\text { Width: } 16 \mathrm{px} .\end{array}$ & $\begin{array}{l}\text { Este icono representa el } \\
\text { objetivo }\end{array}$ \\
\hline Conocimiento previo & :-1 & $\begin{array}{l}\text { Height: 16px. } \\
\text { Width: } 16 \mathrm{px} .\end{array}$ & $\begin{array}{l}\text { Este icono representa los } \\
\text { conocimientos previos }\end{array}$ \\
\hline Actividad de lectura & Eل & $\begin{array}{l}\text { Height: 16px. } \\
\text { Width: 16px. }\end{array}$ & $\begin{array}{l}\text { Este icono representa las } \\
\text { actividades de lectura. }\end{array}$ \\
\hline Reflexión & - & $\begin{array}{l}\text { Height: 16px. } \\
\text { Width: 16px. }\end{array}$ & $\begin{array}{l}\text { Este icono representa la } \\
\text { reflexión. }\end{array}$ \\
\hline Actividad & 家 & $\begin{array}{l}\text { Height 16px. } \\
\text { Width: 16px. }\end{array}$ & $\begin{array}{l}\text { Este icono representa la } \\
\text { actividad. }\end{array}$ \\
\hline
\end{tabular}

Figura 7 Estandarización de iconos

Fuente: Elaboración Propia

\section{c) Diseño físico}

En este proceso se definieron las características físicas de la aplicación, la presentación y visualización de los elementos de instrucción, secuencias y utilización de multimedios, en este apartado se lleva a cabo la descripción de los contenidos, el uso de colores, video, y gráficos.

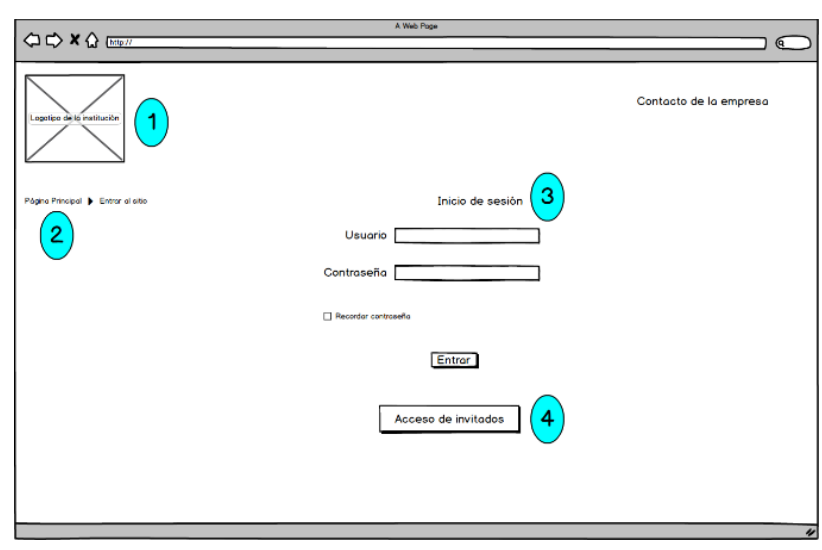

Figura 8 En la siguiente figura se muestran los apartados de acceso a la plataforma virtual

Fuente: Elaboración Propia

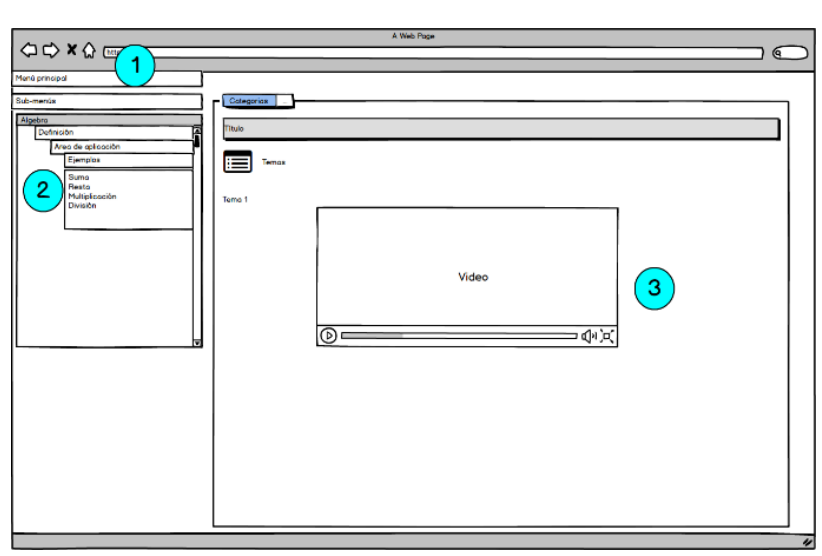

Figura 9 En la siguiente figura se muestran los apartados de acceso las secciones de distribución de los recursos didácticos y contenidos en la plataforma virtual Fuente: Elaboración Propia

\section{Barra superior}

a) Menú principal. Conformado por archivo, unidades, estilos y ayuda.

2. En la barra lateral Izquierda.

a) Submenú. En este apartado se desplegará el nombre de la materia, el tema, área de aplicación y ejemplos

3. En la parte central 
a) Videos cargados. Se ubicarán los videos que estarán en reproducción.

\section{Fase III. Desarrollo}

En esta fase con el uso software se desarrollaron herramientas multimedia como infografías, videos basados en guiones y exámenes de opción múltiple con retroalimentación para los estudiantes.

a) Herramientas de desarrollo:

Las imágenes fueron diseñadas en Ilustrator CS6, los videos en Wondershare Filmora9, ExeLearning para crear contenidos para la web, balsamiq facilitó la creación de prototipos

b) Incorporación de multimedios.

En la incorporación de multimedios se desarrollaron infografía, videos, y exámenes en línea.

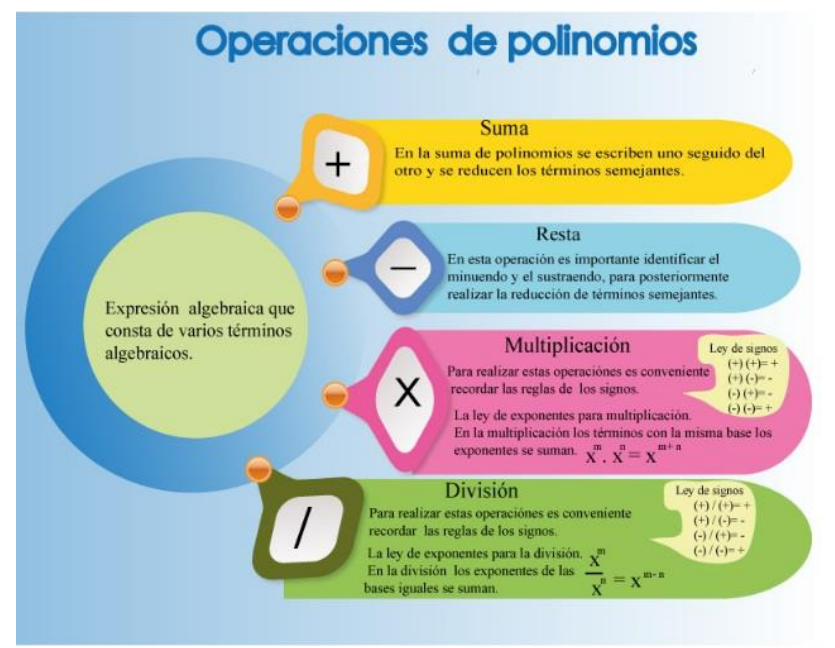

Figura 10 En la siguiente infografía diseñada se describe el concepto de las operaciones básicas de polinomios que son, suma, resta, multiplicación y división

Fuente: Elaboración Propia

\section{Fase IV: Implantación, producción y entrenamiento}

Para su implantación se integraron los recursos multimedios a la Plataforma UTHH Virtual para poder realizar la primera prueba de utilización. Esta fase se desarrollará en una segunda etapa del proyecto ya que se brindará capacitación a docentes y alumnos para el acceso a la plataforma. Se realizará una prueba piloto para evaluar aspectos funcionales, técnicos, estéticos, expresivos, pedagógicos y de eficiencia.

\section{Resultados}

Para determinar los tipos de recursos didácticos a desarrollar se consideró el análisis de la secuencia didáctica de los módulos para que los alumnos puedan repasar temas vistos en clase. Por lo que la selección de temas se basó en hoja de asignatura de la materia de algebra lineal y resultados de diagnóstico de aritmética y algebra a alumnos de nuevo ingreso (UTHH, INSTRUMENTOS DE EVALUACIÓN, 2019)

a) La academia de matemáticas determino la materia, los temas para iniciar el proceso de desarrollo de recursos didácticos, por lo que se realizó un mapeo de las competencias y las materias relacionadas a las matemáticas de los diez programas educativos que oferta la Universidad a nivel Técnico Superior y diez programas educativos nivel licenciatura. Se determinó que cada tema tendría una definición, campo de aplicación, ejemplos, practicas, evaluación y bibliografía. Para la selección de medios de enseñanza en relación con los sistemas de comunicación se consideró si estos eran escritos, visuales, escritos y visuales, auditivos, audiovisuales, interactivos, si tenían sincronía y si podrían presentarse a distancia.

b) Se desarrollaron guiones para determinar la secuencia y contenidos de los videos; en la siguiente figura se muestra el procedimiento de la multiplicación de un monomio por monomio 


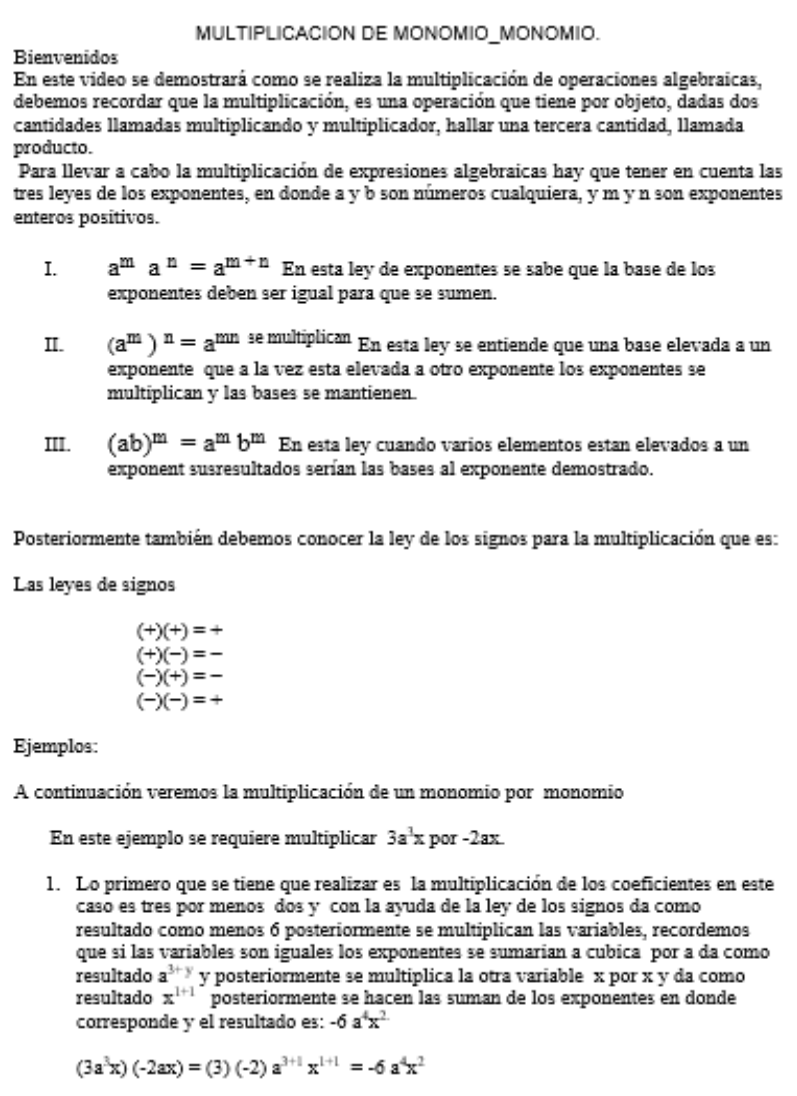

Figura 11 En la siguiente figura es un guion donde se explica el procedimiento para realizar la multiplicación de un monomio por monomio

Fuente: Elaboracón Propia

c) En el desarrollo de recursos didácticos se consideró que "La interactividad debe entenderse de forma que propicie una interacción tanto con los contenidos y materiales de información, tanto horizontal y vertical, con todos los participantes en el mismo, sean estos profesores alumnos o administradores del sistema. (Barroso \& Cabrero, 2000) Como resultado se diseñaron e incorporaron multimedios desarrollando videos interactivos basados en guiones $\mathrm{y}$ secuencias de aprendizaje y en la plataforma se desarrollaron test de elección múltiple con retroalimentación para los estudiantes.

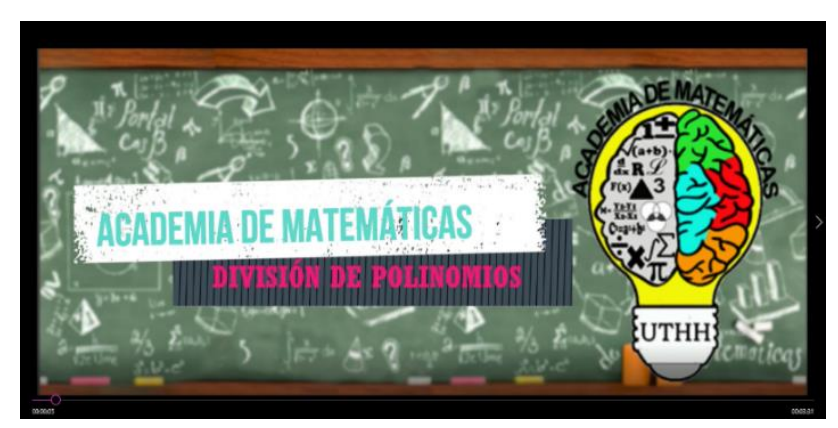

Figura 21 Eta imagen representa el desarrollo del video "división de polinomios"

Fuente: Elaboracón Propia

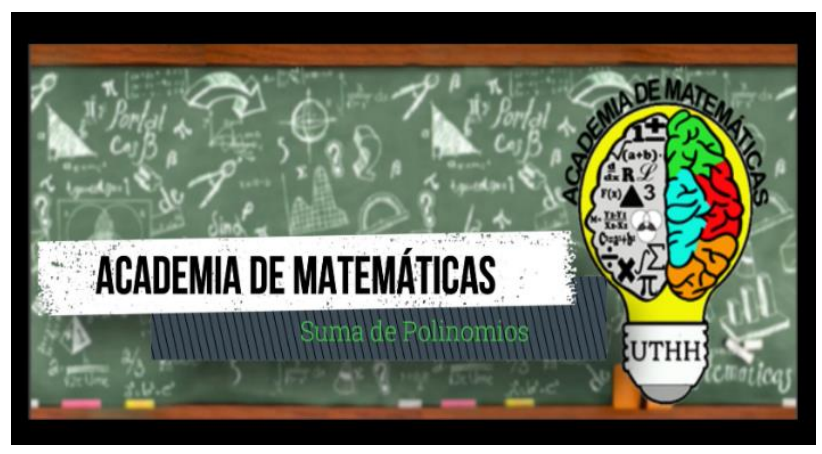

Figura 13 Eta imagen representa el desarrollo del video "suma de polinomios"

Fuente: Elaboracón Propia

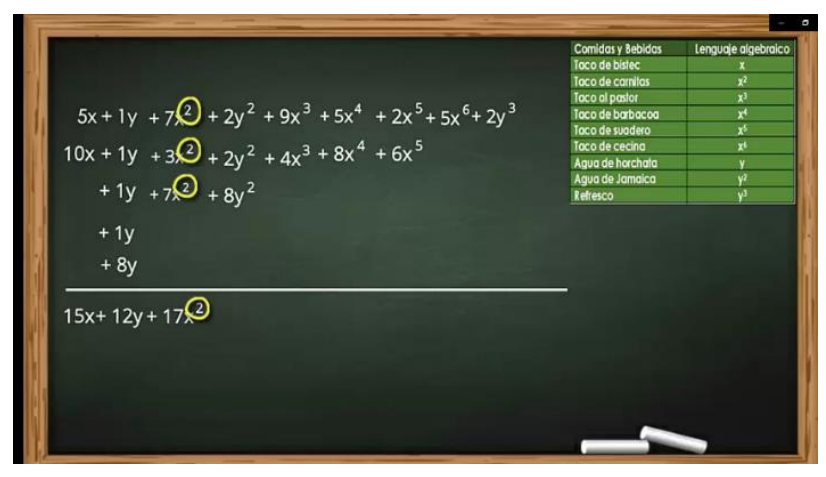

Figura 14 Eta imagen representa el desarrollo del video "suma de polinomio donde se muestra el proceso de solución"

Fuente: Elaboracón Propia

d) En la plataforma virtual UTHH se subieron los recursos didácticos, que estarán disponibles para la comunidad universitaria como un proyecto de asesorías, que apoye el proceso de enseñanza aprendizaje.

En trabajos futuros se pretende realizar la capacitación a los docentes para la incorporación de recursos didácticos; y alumnos para el acceso a la plataforma virtual; además de la incorporación de un foro donde los alumnos puedan debatir los temas y resolver sus dudas en grupo dirigidos.

\section{Agradecimientos}

Se agradece toda la atención, participación de la academia de matemáticas UTHH, al alumnado de con su apoyo y conocimientos fortalecieron el desarrollo del proyecto, a todos los integrantes del Cuerpo Académico en Tecnologías de la información y comunicación por su colaboración en este proyecto que permitirá brindar asesorías a los estudiantes de la Universidad Tecnológica de la Huasteca Hidalguense.

DEL CARMEN-MORALES, Yucels Anaí, DEL CARMEN-MORALES Heidi, FELIPE-REDONDO, Ana María y HERNÁNDEZ-BAUTISTA, Anayeli. Desarrollo de recursos didácticos con el uso de las Tecnologías de la información y comunicación disponibles en una plataforma virtual. Revista de Tecnologías de la Información. 2019 


\section{Referencias}

Barroso, J., \& Cabrero, J. (2000). Principios para el diseño de materiales multimedoa educativos en la red. En AGUADED, J. y CABERO, J. Educar en red. Málaga: Aljibe.

Bianchini. (1992). Metodologia Desarrollo de Aplicaciones educativas en ambientes multimedia. Obtenido de http://www.ldc.usb.ve/ abianc/mmm.html

Cañedo, C., \& Cáceres, M. (2014). FUNDAMENTOS TEÓRICOS PARA LA IMPLEMENTACIÓN DE LA DIDÁCTICA EN EL PROCESO ENSEÑANZA-APRENDIZAJE. Obtenido de https://docs.google.com/file/d/0B1pw8VI9o8mZjRLMjdVX1djVG8/edit

Castro, M., \& Colmenar, A. (s.f.). Tecnológia Multimedia. Obtenido de https://ocw.innova.uned.es/mm2/tm/contenidos/ pdf/tema4/tmm_tema4_video_digital.pdf

Miranda, A. (2004). De los Ambientes virtuales de aprendizaje a las comunidades de aprendizaje en línea. Revista Digital Universitaria UNAM, 1- 15 .

Ponce, H. (2006). Enseñar y aprender matematicas. Buenos Aires: Centro de Publicaciones Educativas y Material Didàctico.

Spiegel, A. (2006). RECURSOS DIDACTICOS. Buenos Aires: Banco Interamericano de Desarrollo.

Stiles, M. (2000). Effective Learning and the Virtual Learning Environment ISBN 83913639 1 0. Citeseer: En: EUNIS 2000 Towards Virtual Universities, Instytut Informatyki Politechniki Poznanskiej, http://citeseerx.ist.psu.edu/viewdoc/summary?d oi=10.1.1.616.2633.

UTHH. (2019). Estadistica basica institucional Obtenido

de http://sgi.uthh.edu.mx/rrectores/IPL/EstBasica_ Inst.php

UTHH. (2019). INSTRUMENTOS DE EVALUACIÓN. Obtenido de http://sgi.uthh.edu.mx/rrectores/IPL/index.php
Rico, Sierra, \& Castro. (2000). DIDÁCTICA DE LAS MATEMÁTICAS Y EDUCACIÓN MATEMÁTICA. London: Lawrence Erlbaum Ass: International handbook of mathematics education.

S/N. (s.f.). Infografias. Obtenido de https://ideasdeclases.files.wordpress.com/2017/ 02/lista-de-cotejo-para-evaluarinfografc3adas.pdf

UTHH. (2009). Oferta Educatica. Obtenido de http://uthh.edu.mx/2015/?val=b2ZlcnRhX2Vkd WNfdHN1LnBocA==\&M=Mg== 